\title{
New Developments in Automated Particle Analysis in the Electron Microscope - from Micro to Nano
}

\author{
Christian Lang ${ }^{1}$, Matthew Hiscock ${ }^{1}$, James Holland ${ }^{1}$, Susumu Yamaguchi ${ }^{2}$, David Joyce, ${ }^{3}$ and Georgia \\ Vatougia $^{3}$ \\ 1. Oxford Instruments Nanoanalysis, High Wycombe UK \\ 2. Oxford Instruments KK, Tokyo, Japan. \\ 3. Mantis Deposition Ltd., Thame, UK
}

As EDS detectors have become faster, computers more powerful and data storage space cheaper, EDS analysis has become more image centric. X-ray mapping is now used routinely and can be combined with automated stage movements and stitching of maps, so that large sample areas can be covered at high resolution. These very large data sets contain a wealth of information which so far has often not been fully utilized. A new Particle Analysis software (AZtecFeature) enables the automated processing of these large data sets in order to identify features of interest in the sample and measure their morphology and composition. As in conventional particle analysis, the electron image is used to identify the location of features. Different to conventional particle analysis, the new particle analysis software has the option to link a set of electron images to a set of EDS maps of the same area, from which it extracts the compositional information. As these maps can cover the whole sample area, the new particle analysis system can store a virtual sample (figure 1a). This virtual sample can be analyzed offline (figure $1 \mathrm{~b})$, enabling the user to adjust threshold settings and also to investigate features which span more than one field of view. Storing and working with virtual samples enables their sharing amongst research groups over the internet, avoiding the need for transportation of rare or precious samples.

In order to increase data throughput, the new system can acquire data from multiple EDS detectors. In addition to increasing data throughput, we show how multiple detectors can also be used to minimize shadowing of smaller particles by larger ones if the detectors are mounted on opposite ports on the SEM. Figure 2a shows an X-ray map acquired with one EDS detector overlaid over an electron image of a large particle with several smaller particles surrounding it. The EDS detector is positioned on the left hand side of the image, which results in shadowing of some of the smaller particles to the right hand side of the image. Figure $2 \mathrm{~b}$ shows the same particles, now mapped with two detectors mounted on opposing ports; shadowing is clearly eliminated.

Developments in both the electron microscope and EDS detector hardware enable the acquisition of compositional data from particle sizes down to a few nanometers. Very high solid angle detectors on either a TEM or an Ultra-High Resolution SEM such as the Hitachi SU9000 enable the elemental mapping of particles down to $2 \mathrm{~nm}$ in size (figure 3a inset). While the X-ray map clearly shows small particles on the scale of a few nanometers, measuring their size accurately is difficult due to noise in the map. Using automated particle analysis we can correlate the electron image with an EDS map of the area. This way, the particle size distribution can be measured from the less noisy, higher resolution electron image (figure 3). The particle size distribution plotted as a function of the equivalent circular diameter in figure 3 shows that particles below $5 \mathrm{~nm}$ are clearly resolved. The location of the particles can be correlated to the EDS map shown in the inset. As every pixel in the electron image is correlated to a full X-ray spectrum in the EDS map, the software can reconstruct the X-ray spectrum for each particle in order to confirm the particle composition. 

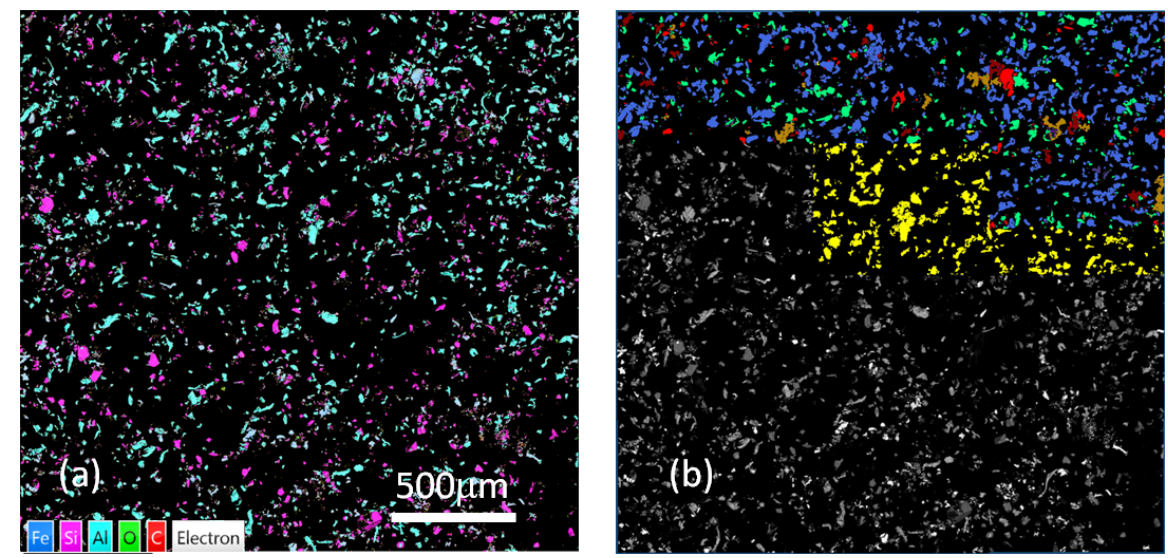

Figure 1. (a) shows a large area map of wear particles containing 12 fields of view. (b) shows the extraction and classification of particle data from the maps in progress.
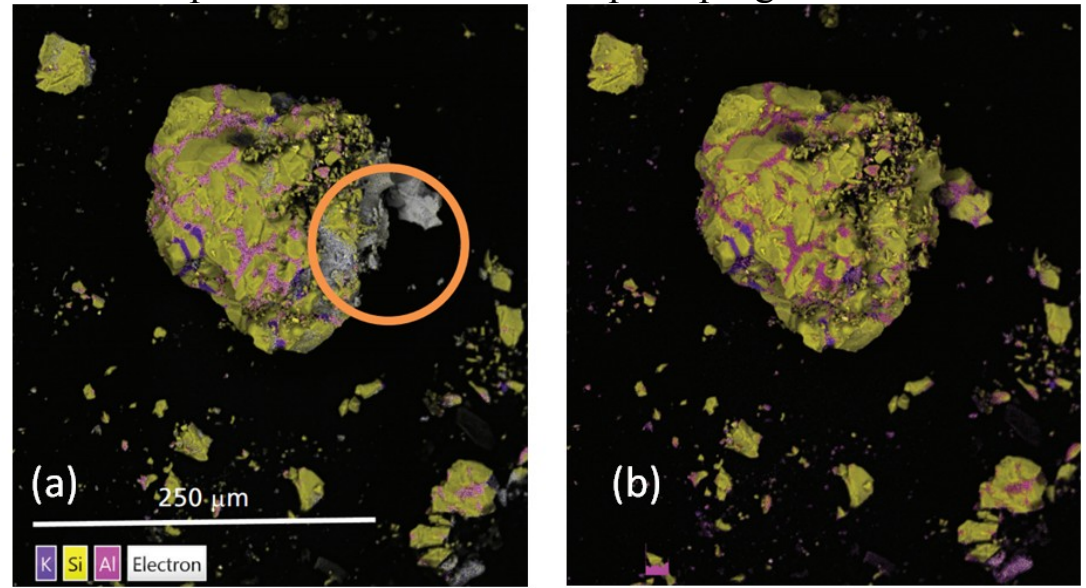

Figure 2. (a) one EDS detector on the left side of the images leads to shadowing in the area circle. (b) shows the same area now with two EDS detectors mounted left and right.

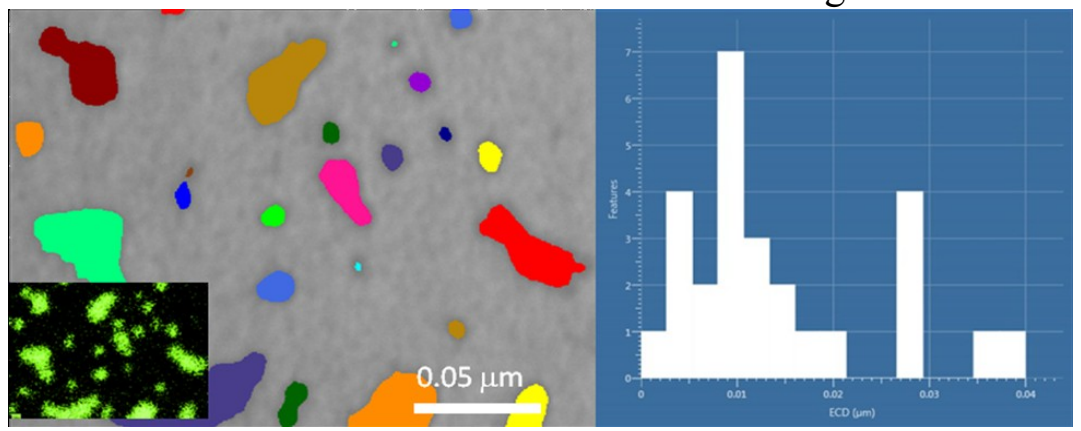

Figure 3. Electron image of small Molybdenum particles down to $2 \mathrm{~nm}$ size (Mo-L map in the inset). Particle have been detected in the electron image (colored). The particle size distribution is shown in the histogram (right). 